\title{
Equidade ameaçada: Assimetrias regionais nos investimentos em medicamentos no
}

\author{
Brasil \\ Threatened equity: Regional asymmetries in drug investments in Brazil \\ Equidad amenazada: asimetrías regionales en inversiones en medicamentos en Brasil
}

Recebido: 22/09/2021 | Revisado: 02/10/2021 | Aceito: 06/10/2021 | Publicado: 09/10/2021

Wendell Rodrigues Oliveira da Silva
ORCID: https://orcid.org/0000-0002-7967-9962
Ministério da Saúde, Brasil
Universidade de Brasília, Brasil
E-mail: wendelloliveir@ gmail.com
Rafael Santos Santana
Universidade de Brasília, Brasil
ORCID: https://orcid.org/0000-003-4481-10X
E-mail: rafaels.santana1@ gmail.com
Anna Maly de Leão e Neves Eduardo
ORCID: https://orcid.org/0000-0003-2861-4402
Faculdade Unyleya, Brasil
Faculdade Anhanguera de Brasília, Brasil
E-mail: annamaly07@ @otmail.com
Rayssa Samara Ferreira Martins
ORCID: https://orcid.org/0000-0002-6426-0878
Faculdade Anhanguera de Brasília, Brasil
E-mail: Rayssasamarafm @gmail.com
Silvana Nair Leite
ORCID: https://orcid.org/0000-0002-5258-9684
Universidade Federal de Santa Catarina, Brasil
E-mail: silvana.nair@ @otmail.com

\section{Resumo}

Os sistemas de saúde dos países estão sofrendo aumento nos seus gastos, por vários motivos, no Brasil não é diferente. De 2006 a 2013 os investimentos com compras de medicamentos pelo Governo Federal aumentaram $172 \%$. Apesar das conquistas alcançadas com o SUS, o acesso a medicamentos ainda não é fácil em várias regiões brasileiras. Nesse trabalho foi realizada a análise dos investimentos em medicamentos pela União, estados e municípios entre 2010 e 2019, objetivando identificar as diferenças regionais na aplicação desses recursos. Observouse que apesar dos investimentos per capta em saúde ter sido maior pelos municípios ( $\$$ \$926,54), quando se trata de medicamentos esse investimento per capta é maior pela União (R \$ 61,89). Identificou-se também que os municípios das regiões Nordeste e Norte investem menos na aquisição de medicamentos quando comparado com a média nacional ( $\mathrm{R} \$ 40,41), \mathrm{R} \$ 24,20$ e $\mathrm{R} \$ 23,58$ respectivamente. Ao explorar o percentual de investimento em medicamento frente ao investimento total em saúde entre os entes, é possível verificar que estados e municípios vêm diminuindo seus percentuais de investimento em medicamentos, destacando-se a maior redução pelos estados $(72,8 \%$ durante o período avaliado). Quando utilizado indicador de vulnerabilidade, consta-se que quanto maior a vulnerabilidade social do município, menores são os valores investidos em medicamentos. Os dados demonstram que pode haver comprometimento na execução da oferta de medicamentos pelos principais entes assistenciais (municípios) e especialmente nas regiões com maior grau de vulnerabilidade social, justamente onde mais se depende do apoio do serviço público de saúde.

Palavras-chave: Investimento em saúde; Serviços de assistência farmacêutica; Custos de medicamentos; Política Nacional de Assistência Farmacêutica.

\begin{abstract}
The health systems of the countries are suffering an increase in their expenses, for several reasons in Brazil, it is not different. From 2006 to 2013, investments in drug procurements by the Federal Government increased by $172 \%$. Despite the achievements with the SUS, access to medicines is still not easy in several Brazilian regions. This work aims to analyze investments in medicines made by the Union, states and municipalities between 2010 and 2019 in order to identify regional differences in the application of these financial resources. It was observed that although the per capita investments in health were higher by the municipalities ( $\mathrm{R} \$ 926.54)$, when it comes to medicines, this per capita investment is higher by the Union ( $\mathrm{R} \$ 61.89)$. It was also identified that municipalities in the Northeast and North regions invest less in the procurement of medicines when compared to the national average (R\$40.41), R\$ 24.20 and $\mathrm{R} \$ 23.58$ respectively. When exploring the percentage of investment in medicine compared to the total
\end{abstract}


investment in health among entities, it is possible to verify that states and municipalities have been decreasing their percentage of investment in medicines, highlighting the largest reduction made by the states $(72.8 \%$ during the period analyzed). When the vulnerability indicator is used, it appears that the greater is the social vulnerability of the municipality, the lower are the amounts invested in medicines. The data show that the supply of medicines may be adversely affected in the main care entities (municipalities), especially in regions with a higher degree of social vulnerability. It is observed precisely where the support of the public health service is most dependent.

Keywords: Investments; Pharmaceutical services; Drug costs; National Policy of Pharmaceutical Assistance.

\section{Resumen}

Los sistemas de salud de los países están sufriendo un aumento en sus gastos, por varias razones, en Brasil no es diferente. De 2006 a 2013, las inversiones en compra de medicamentos por parte del Gobierno Federal aumentaron un $172 \%$. A pesar de los logros alcanzados con el SUS, el acceso a los medicamentos aún no es fácil en varias regiones brasileñas. En este trabajo se realizó un análisis de las inversiones en medicamentos por parte de la Unión, estados y municipios entre 2010 y 2019, con el objetivo de identificar diferencias regionales en la aplicación de estos recursos. Se observó que si bien las inversiones per cápita en salud fueron mayores por parte de los municipios (R\$ 926,54), en lo que respecta a medicamentos, esta inversión per cápita es mayor por parte de la Unión (R\$ 61,89). También se identificó que los municipios de las regiones Nordeste y Norte invierten menos en la compra de medicamentos en comparación con el promedio nacional ( $\mathrm{R} \$ 40,41), \mathrm{R} \$ 24,20$ y $\mathrm{R} \$ 23,58$ respectivamente. Al explorar el porcentaje de inversión en medicina frente a la inversión total en salud entre entidades, se puede constatar que los estados y municipios han venido disminuyendo su porcentaje de inversión en medicamentos, destacando la mayor reducción por estados (72,8\% durante el período evaluado). Cuando se utiliza el indicador de vulnerabilidad, parece que cuanto mayor es la vulnerabilidad social del municipio, menores son los montos invertidos en medicamentos. Los datos muestran que las principales entidades asistenciales (municipios) pueden verse comprometidas en el suministro de medicamentos, y especialmente en regiones con mayor grado de vulnerabilidad social, precisamente donde el apoyo del servicio público de salud es más dependiente.

Palabras clave: Inversiones en salud; Servicios farmacéuticos; Costos de los medicamentos; Política Nacional de Asistencia Farmacéutica.

\section{Introdução}

Os governos mundiais têm enfrentado há décadas um dos maiores desafios, que é o de gerenciar os gastos com diferentes serviços de saúde, e os aumentos com os gastos em saúde são multifatoriais (Kondro, 2007). O envelhecimento populacional e o aumento dos gastos com medicamentos novos, por exemplo, imprimem uma pressão considerável nos esforços dos sistemas de saúde para continuar fornecendo cuidados abrangentes.

O Brasil, como outros países de renda média em crescimento, experimenta um aumento da prevalência de doenças crônicas na população associado a permanência de muitas doenças transmissíveis agudas, gerando uma sobreposição de cargas de doença que ampliam a demanda de gastos com serviços de saúde e medicamentos, o que só agrava os riscos para a sustentabilidade dos orçamentos públicos periodicamente constrangidos pelas medidas de austeridade fiscal (Massuda et al., 2018).

Paim et al. (2011) demonstram que avanços foram feitos na implantação do Sistema Único de Saúde (SUS). Inovações institucionais foram introduzidos, incluindo uma descentralização substancial do processo que concedeu maior responsabilidade aos municípios para gestão de serviços de saúde e meios pelos quais potencializa e formaliza a participação social em saúde em formulação de políticas públicas e responsabilidade. Apesar de todos os avanços alcançados, o SUS é um sistema de saúde em contínuo desenvolvimento que ainda está lutando para possibilitar a cobertura equitativa.

No SUS, as aquisições de medicamentos são realizadas pela União, pelos 26 estados e Distrito Federal e pelos 5.570 municípios (Vieira \& Zucchi, 2011). Entre 2000 e 2013, os medicamentos foram responsáveis por 19\% a 22,4\% das despesas com saúde no Brasil (Dias et al., 2019) e no total, estimasse que o gasto público com medicamentos nas três esferas de governo passou, em termos reais, de R \$ 14,3 bilhões em 2010 para R \$ 18,6 bilhões em 2016 (Vieira, 2018).

De acordo com os dados do Sistema Integrado de Administração de Serviços Gerais (SIASG), de 2006 a 2013 só no governo federal os gastos com compras de medicamentos aumentaram 172\% (Luz et al., 2017). 
Apesar do incremento dos investimentos nessa área desde a criação do SUS, a falta de acesso aos medicamentos ainda é uma realidade, mesmo se tratando de um direito constitucional (Castro et al., 2019; Luiza et al., 2018; Nascimento, J. Álvares, et al., 2017). Verificava-se em 2014, nas unidades de saúde do sistema público de saúde brasileiro, não mais que 62\% de disponibilidade de medicamentos essenciais, apesar dos esforços do Brasil para alcançar a cobertura universal (Garcia et al., 2020).

Costa (2014) demonstrou em sua pesquisa que os maiores percentuais de hipertensos e diabéticos que precisaram comprar medicamento em farmácias e drogarias concentraram-se na região Nordeste e os menores percentuais foram observados na região Sudeste. Drummond et al. (2020) trouxeram que a prevalência de não adesão à farmacoterapia no Brasil, segundos dados da PNAUM, foi de $20,2 \%$, sendo as regiões Nordeste $(27,8 \%)$ e Norte $(24,2 \%)$ as com maiores prevalências. E Nascimento et al., (2017), ainda com base em dados da PNAUM, falam que a disponibilidade geral de medicamentos variou conforme as regiões do país, sendo as regiões Norte $(44,6 \%)$ e Nordeste $(46,3 \%)$ as com menores disponibilidade.

Oliveira et al. (2016) concluíram que a acessibilidade geográfica às farmácias de medicamentos para doenças crônicas não transmissíveis pode melhorar, especialmente para as farmácias do SUS e farmácias do Programa Farmácia Popular nas regiões Norte, Nordeste e Centro-Oeste.

Corrobora com o citado anteriormente o fato que inúmeras ações judiciais movidas pelos cidadãos no Brasil objetivam contornar a falta de medicamentos para tratamentos de doenças diversas como câncer e doenças genéticas, que não estão inclusas na política de Assistência Farmacêutica ou que não atendem as suas expectativas (Chagas et al., 2020; Wang et al., 2014). Nos milhares de casos de judicialização, o medicamento é o campeão de pedidos dos pacientes na justiça (Reveiz et al., 2013; Vargas-Pelaez et al., 2019).

Mendes et al. (2020) ao proporem o índice de necessidades de saúde entre os estados brasileiros já evidenciam a desigualdade de saúde entre as regiões brasileiras, concentrando os maiores valores do índice nas regiões Norte e Nordeste e os menores nas regiões mais ao sul do país, além do Distrito Federal.

Para a obtenção dos dados dessa pesquisa utilizou-se dados do Sistema de Informações Sobre Orçamentos Públicos em Saúde (SIOPS) que está disponível através do Departamento de Informática do SUS (DATASUS) no Portal da Saúde e é calculado utilizando vários indicadores dos gastos de cada município, dentre eles: a receita de impostos e transferências constitucionais legais per capita; o percentual da receita de impostos próprios sobre a receita de impostos e transferências constitucionais legais; o percentual da receita do fundo de participação dos municípios (FPM) sobre a receita de impostos e transferências constitucionais legais; o percentual da receita do imposto sobre circulação de mercadorias e prestação de serviços (ICMS) sobre a receita de impostos e ainda as transferências constitucionais legais (Santos Neto et al., 2017).

Reconhecer os investimentos em medicamentos como componente essencial na garantia do acesso à saúde é fundamental. No entanto, as desigualdades econômicas e sociais no Brasil, e a organização descentralizada da assistência farmacêutica indicam para a necessidade de analisar os investimentos realizados pelos entes federados em saúde e medicamentos, e identificar assimetrias regionais na aplicação desses recursos que, em última análise, representam impactos na oferta e acesso a medicamentos pelo SUS.

\section{Metodologia}

Trata-se de um estudo exploratório, retrospectivo, no qual verifica-se a evolução dos investimentos em saúde e em medicamentos pela União, estados, Distrito Federal e municípios.

Os dados foram coletos do Sistema de Informação sobre Orçamentos Públicos em Saúde (SIOPS) (Ministério da Saúde, 2017) que constitui instrumento para o acompanhamento do cumprimento do dispositivo constitucional que determina, 
em orçamento, a aplicação mínima de recursos em ações e serviços públicos de saúde. Os indicadores utilizados foram os relacionados a investimento em saúde e em medicamentos dos três entes, dos anos de 2010 a 2019.

Extraiu-se do SIOPS e analisou-se os dados da União, dos estados e dos municípios da amostra referentes a:

I. despesa total em saúde (D.Total Saúde), que representa os gastos totais com saúde dos entes durante o período avaliado;

Esse indicador resulta do gasto em saúde, por habitante, advindo de todas as fontes, quer sejam impostos, transferências do SUS (União, Estados e outros Municípios), operações de créditos e outras.

II. e a participação percentual da despesa com medicamentos na despesa total com saúde que representam a participação dos gastos com medicamentos em relação ao total dos gastos com Saúde (\%D.com Medicamentos).

Ressalta-se que as despesas com medicamentos realizadas por meio do SUS (Hospitais e Postos de Saúde) compõem o indicador supracitado, entretanto, aquelas realizadas com características de assistência social a pessoas de baixa renda (distribuição de medicamentos) não compõem o cálculo deste indicador.

Como a natureza do sistema é auto declaratória, e por não ser o foco do trabalho, não foi possível identificar quais os valores das despesas dos estados foram pagos com recursos próprios ou com recursos advindos da União, e em relação as despesas dos municípios, quais foram quitadas com recursos oriundos da União, do Estado ou do próprio ente.

Foram calculados os valores per capta de acordo com a população abrangente de cada ente. Os valores foram corrigidos para janeiro de 2020 pelo Índice de Preços ao Consumidor Amplo - IPCA (Banco Central do Brasil, n.d.).

Os dados da população utilizada de 2010 a 2019 para cálculo dos valores per capta dos entes da pesquisa foram extraídos também do SIOPS (Ministério da Saúde, 2017). Esses dados veem da base do IBGE, base essa que é alimentada pelo censo e suas estimativas anuais.

Além dos dados referentes à União, analisou-se 26 unidades federativas, pois o Distrito Federal foi excluído e Brasília foi incluída nos municípios, isso para evitar duplicidade dos dados, e foram selecionados 600 municípios brasileiros, para análise dos entes municipais. Para fins comparativos, reproduziu-se a amostra da Pesquisa Nacional Sobre o Acesso, Utilização e Promoção do Uso Racional de Medicamentos no Brasil (PNAUM), maior pesquisa brasileira da área. A amostra composta por 120 municípios em cada uma das 5 regiões brasileiras, selecionados da seguinte forma (Álvares, Alves, et al., 2017):

- $\quad$ capitais $(4,5 \%)$;

- municípios maiores, tendo sido tomados os 0,5\% maiores municípios da região, de acordo com a população (4,5\%), e;

- municípios menores, com sorteio sistemático, também de acordo com o tamanho da população, dentro de cada região $(91 \%)$.

Trata-se de uma amostra ponderada e dá a condição de responder, com confiabilidade, o que acontece em cada região do país (Quadro 1). A fração de amostragem correspondente ao sorteio de municípios, em cada estrato, foi: $f=a / A$, sendo $a$ o número de municípios na amostra e $A$ o número de municípios existentes. Nos dois primeiros estratos (de capitais e de municípios maiores) a fração de amostragem correspondeu à unidade (Álvares, Alves, et al., 2017). 
Quadro 1. Porte dos municípios por região.

\begin{tabular}{|l|l|l|l|l|}
\hline Regiões & Capital & $\begin{array}{l}\text { Municípios } \\
\text { maiores* }\end{array}$ & $\begin{array}{l}\text { Demais } \\
\text { municípios** }\end{array}$ & Total \\
\hline Norte & 7 & 2 & 111 & 120 \\
\hline Sul & 3 & 6 & 111 & 120 \\
\hline Nordeste & 9 & 9 & 102 & 120 \\
\hline Sudeste & 4 & 8 & 108 & 120 \\
\hline Centro-oeste & 4 & 2 & 114 & 120 \\
\hline TOTAL & $\mathbf{2 7}$ & $\mathbf{2 7}$ & $\mathbf{5 4 6}$ & $\mathbf{6 0 0}$ \\
\hline
\end{tabular}

* Considerando o tamanho da população

**Escolhidos por sorteio sistemático entre os municípios com menor população dentro da região

Fonte: Álvares, Alves, et al. (2017)

Utilizou-se, ainda, o Índice de Vulnerabilidade Social (IVS) que, segundo o IPEA (2015) é composto de dezesseis indicadores estruturados em três dimensões: Infraestrutura Urbana, Capital Humano e Renda e Trabalho, e que sinaliza o acesso, a ausência ou a insuficiência de alguns "ativos" em áreas do território brasileiro. O IVS é um índice que varia entre $0 \mathrm{e}$ 1. Quanto mais próximo a 1, maior é a vulnerabilidade social de um município. Para os municípios que apresentam IVS entre 0 e 0,200, considera-se que possuem muito baixa vulnerabilidade social. Valores entre 0,201 e 0,300 indicam baixa vulnerabilidade social. Aqueles que apresentam IVS entre 0,301 e 0,400 são de média vulnerabilidade social, ao passo que, entre 0,401 e 0,500 são considerados de alta vulnerabilidade social. Qualquer valor entre 0,501 e 1 indica que o município possui muito alta vulnerabilidade social (IPEA, 2015).

De posse desses dados, procurou-se analisar a evolução e as assimetrias regionais dos recursos investidos em saúde e em medicamentos pela União, estados e pelos municípios.

\section{Resultados}

Em 2019, o montante investido pela União em saúde e em medicamentos foi de 132 bilhões de reais e 13 bilhões de reais, respectivamente, ao passo que os estados investiram nessas áreas $\mathrm{R} \$ 102$ bilhões e 1,7 bilhão. Os municípios brasileiros investiram no total 168 bilhões de reais na saúde e R 5 bilhões em medicamentos para seus munícipes, segundo dados do SIOPS.

Os investimentos públicos em saúde representam os gastos em ações e serviços de saúde realizados pelos entes públicos para sua população. Pode-se observar pelo Gráfico 1 que os valores de investimento per capta em saúde da União por ano, aumentaram 30,7\% em 2019 quando comparado com 2012. Os dados estaduais demonstram uma pequena queda de investimentos durante o período avaliado e a curva municipal apresentou o maior incremento (32,8\% de 2010 a 2019$)$. 
Research, Society and Development, v. 10, n. 13, e179101320896, 2021

(CC BY 4.0) | ISSN 2525-3409 | DOI: http://dx.doi.org/10.33448/rsd-v10i13.20896

Gráfico 1 - Investimento per capta em saúde por ano.

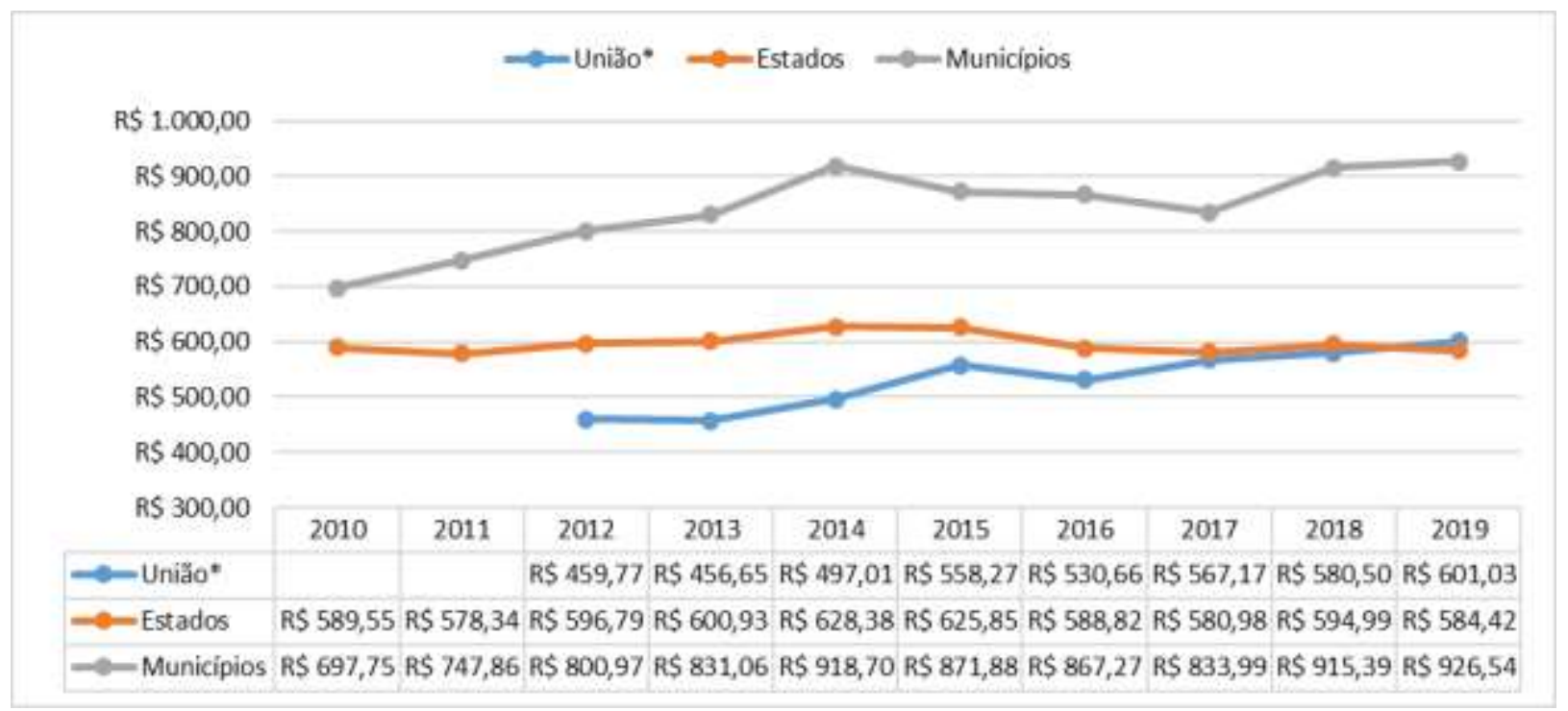

*O SIOPS não apresenta os dados de 2010 e 2011

Valores corrigidos para janeiro de 2020 pelo Índice de Preços ao Consumidor Amplo - IPCA

Fonte: Autores (2021).

Quando se analisa os dados de investimento per capta em saúde dos municípios, por região, de 2010 a 2019 (Gráfico 2), observa-se que houve um aumento em todas as regiões no intervalo e que as regiões Centro-Oeste $(28,61 \%)$, Sudeste $(28,22 \%)$ e Sul $(46,15 \%)$ investiram mais que a média nacional em todo o período, ao passo que as regiões Nordeste e Norte investiram menos que a média nacional, mesmo apresentando crescimentos de $33,77 \%$ e 25,58\%, respectivamente. Apesar do aumento observado entre o início e o fim do intervalo, observa-se que durante o período analisado houve períodos em que o valor per capta em investimento em saúde aumentou em todas as regiões, como de 2010 a 2014, em seguida houve queda em praticamente os municípios de todas as regiões, até 2017, quando esse investimento voltou a subir.

Gráfico 2 - Valores per capta de investimento dos municípios em saúde por ano, por região.

\begin{tabular}{|c|c|c|c|c|c|c|c|c|c|c|}
\hline \multicolumn{2}{|c|}{ —Média Nacional } & \multicolumn{2}{|c|}{$\rightarrow$ Centro-Oeste } & \multicolumn{2}{|c|}{$\longrightarrow$ Nordeste } & \multicolumn{3}{|c|}{$\rightarrow$ Norte } & \multicolumn{2}{|c|}{- Sul } \\
\hline \multirow{7}{*}{$\begin{array}{r}R \$ 1.200,00 \\
R \$ 1.100,00 \\
R \$ 1.000,00 \\
R S 900,00 \\
R S 800,00 \\
R \$ 700,00 \\
R \$ 600,00 \\
\text { RS } 500,00\end{array}$} & \multicolumn{10}{|c|}{$\longrightarrow$} \\
\hline & \multicolumn{10}{|c|}{ 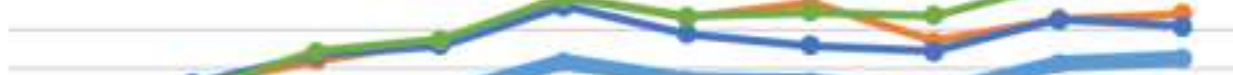 } \\
\hline & \\
\hline & \multirow{2}{*}{\multicolumn{10}{|c|}{$\longrightarrow$}} \\
\hline & & & & & & & & & & \\
\hline & \\
\hline & 2010 & 2011 & 2012 & 2013 & 2014 & 2015 & 2016 & 2017 & 2018 & 2019 \\
\hline$\Rightarrow$ Média Nacional & RS 697,7 & $R \$ 747,8$ & RS 800,9 & RS 831,0 & RS 918,7 & RS 871,8 & RS 867,2 & RS 833,9 & RS 915,3 & RS 926,5 \\
\hline$\rightarrow$-Centro-Oeste & $\mathrm{R} \$ 810,2$ & $\mathrm{R} \$ 856,5$ & $\mathrm{R} \$ 921,3$ & RS 970,9 & $\mathrm{R} \$ 1.087$ & R\$ 1.032 & RS 1.070 & $\mathrm{R} \$ 970,3$ & $R \$ 1.028$ & R\$ 1.042 \\
\hline$\Longrightarrow$ Nordeste & RS 550,5 & $R \$ 599,1$ & RS 627,2 & RS 641,3 & RS 701,1 & RS 677,6 & RS 646,7 & $R \$ 633,6$ & RS 714,2 & RS 736,4 \\
\hline$\longrightarrow$ Norte & $\mathrm{R} \$ 558,6$ & RS 582,7 & RS 575,6 & RS 608,6 & $\mathrm{R} \$ 661,2$ & RS 624,2 & RS 612,8 & $\mathrm{R} \$ 584,8$ & RS 687,3 & RS 701,5 \\
\hline -Sudeste & RS 787,7 & R\$ 861,2 & RS 936,6 & RS 958,7 & $\mathrm{R} \$ 1.061$ & RS 989,7 & RS 960,0 & R\$ 943,6 & R\$ 1.025 & RS 1.010 \\
\hline$\rightarrow$ Sul & RS 781,4 & $\mathrm{R} \$ 839,5$ & R\$ 944,0 & R\$ 975,6 & R\$ 1.082 & R\$ 1.035 & RS 1.045 & $\mathrm{R} \$ 1.037$ & R\$ 1.121 & RS 1.142 \\
\hline
\end{tabular}

Valores corrigidos para janeiro de 2020 pelo Índice de Preços ao Consumidor Amplo - IPCA Fonte: SIOPS. 


\section{Investimento em medicamentos}

O investimento público em medicamentos é importante para ajudar a sanar carências em saúde que acometem grande parte da população brasileira. Nesse trabalho identificou-se os valores investidos em medicamentos pelos 3 entes brasileiros, os valores de investimento dos municípios em medicamento por ano, por região e a percentagem da despesa com medicamentos na despesa em saúde.

Ao se fazer a análise simultaneamente dos valores per capta investidos em medicamentos pela União, estados e municípios, a situação obtida é a observada no Gráfico 3.

Ao analisar os dados de investimento per capta em medicamentos da União, de 2013 a 2019, pode-se identificar que houve um aumento de 23,5\% no valor desse investimento ao longo do período, ao passo que, nos estados, de 2010 a 2019 observa-se que vêm diminuindo esse valor, apresentando no intervalo estudado uma queda de mais de $69 \%$. Os municípios apresentaram um acréscimo de 1,6\% no valor per capta analisado.

Observa-se que a partir de 2014 a União passou a investir em medicamentos bem mais que os demais entes, chegando a apresentar uma diferença em 2019 de 509\% a mais no valor per capta quando comparado com o valor dos estados e 53,2\% quando comparado com os municípios.

Gráfico 3 - Valores per capta de investimento em medicamento pelos três entes.

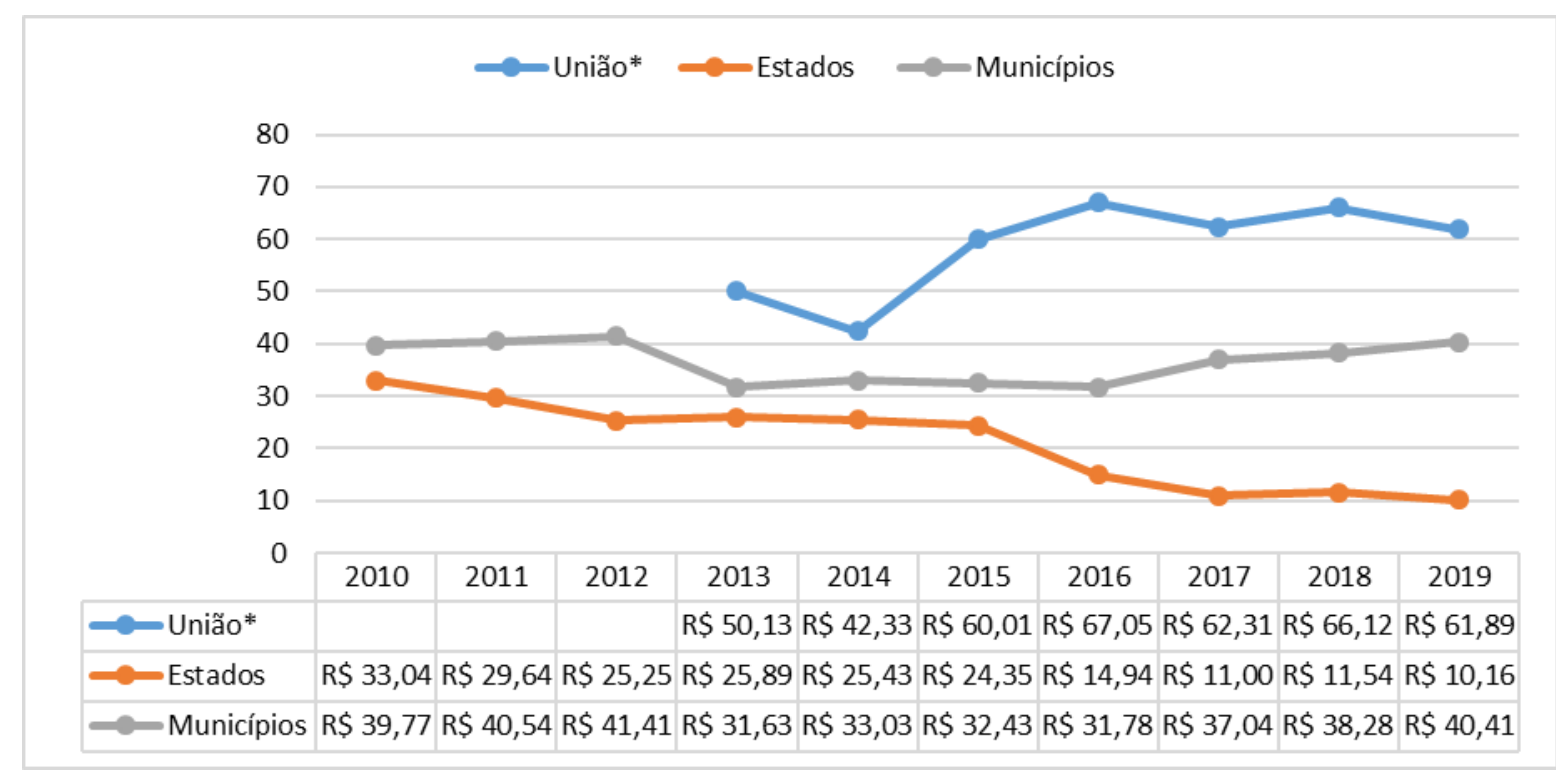

* O SIOPS não apresenta os dados de 2010 a 2012

Valores corrigidos para janeiro de 2020 pelo Índice de Preços ao Consumidor Amplo - IPCA

Fonte: Autores (2021).

Já quando se observa os dados de investimento per capta em medicamentos dos municípios, por região, de 2010 a 2019 (Gráfico 4), é possível observar que nas regiões em que houve aumento no valor per capta (Centro-Oeste, Sudeste e Sul), esse foi muito pequeno. Já as regiões Nordeste e Norte, que em todo o período investiram menos que a média nacional, apresentaram recuo no valor do investimento no intervalo pesquisado de $25,63 \%$ e $21,69 \%$, respectivamente. 
Research, Society and Development, v. 10, n. 13, e179101320896, 2021

(CC BY 4.0) | ISSN 2525-3409 | DOI: http://dx.doi.org/10.33448/rsd-v10i13.20896

Gráfico 4 - Valor per capta de investimento dos municípios em medicamento por ano, por região.

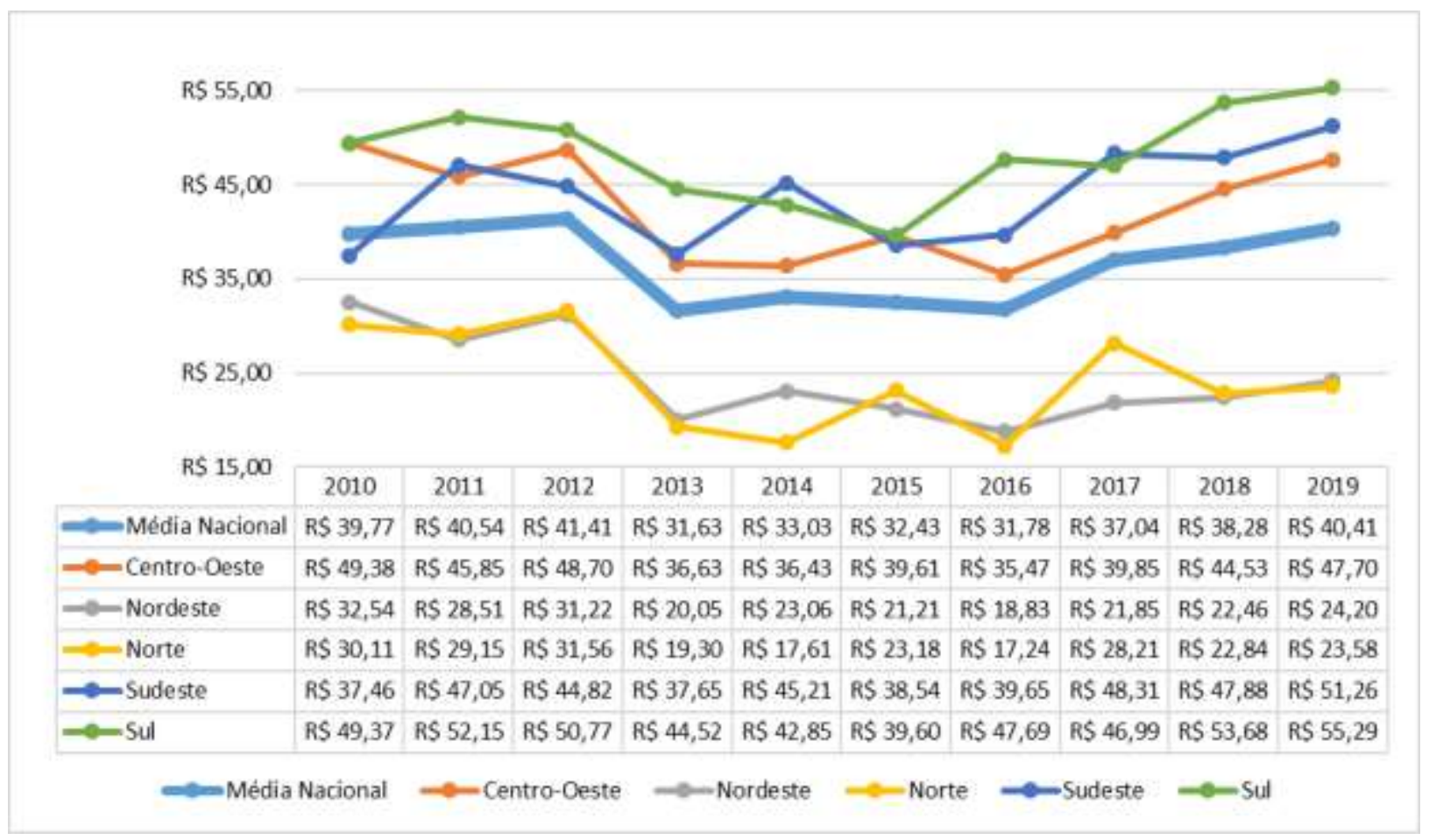

Valores corrigidos para janeiro de 2020 pelo Índice de Preços ao Consumidor Amplo - IPCA.

Fonte: SIOPS.

Ao explorar o percentual de investimento em medicamento frente ao investimento total em saúde entre os entes, é possível verificar um incremento dos valores da União, passando de 7,65\% em 2013 (R\$ 10 bilhões) para 9,84\% em 2019 (R\$ 13 bilhões). Estados e municípios, no entanto, vêm diminuindo seus percentuais de investimento em medicamentos, destacando a maior redução por parte do ente estadual (58,36\%), passando de aproximadamente $\mathrm{R} \$ 339$ milhões para $\mathrm{R} \$ 141$ milhões.

Gráfico 5 - Percentagem de despesa com medicamentos na despesa em saúde.

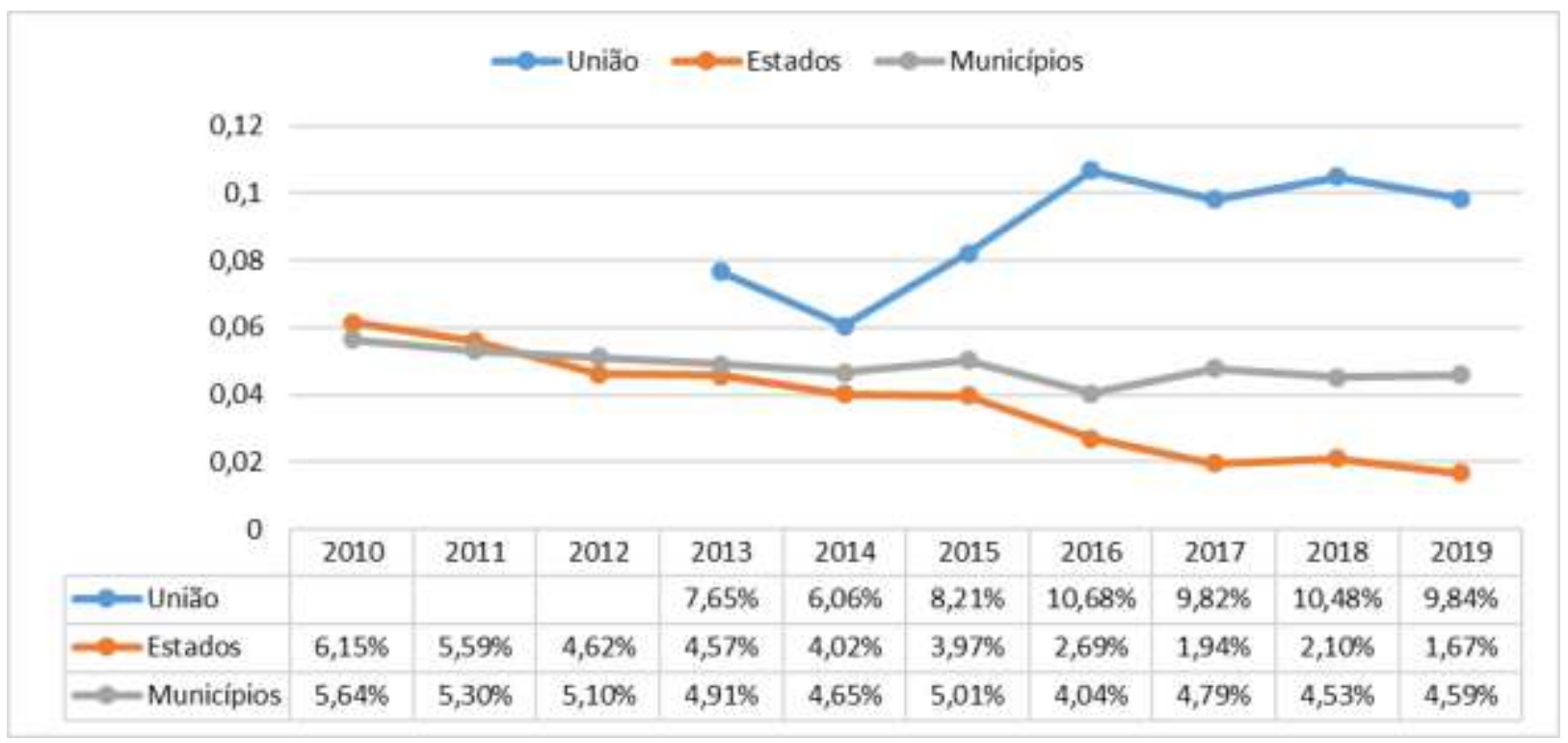

Fonte: Autores (2021). 
O Índice de Vulnerabilidade Social (IVS) é um índice que possibilita aos gestores uma visão das condições de vida de todas as camadas socioeconômicas do país, estados e municípios, identificando àquelas que encontram-se em vulnerabilidade e risco social.

No Gráfico 6 os investimentos em medicamentos são correlacionados com o IVS dos municípios da pesquisa:

Gráfico 6 - Valores per capta investido em medicamento pelos municípios de acordo com a classificação de vulnerabilidade.

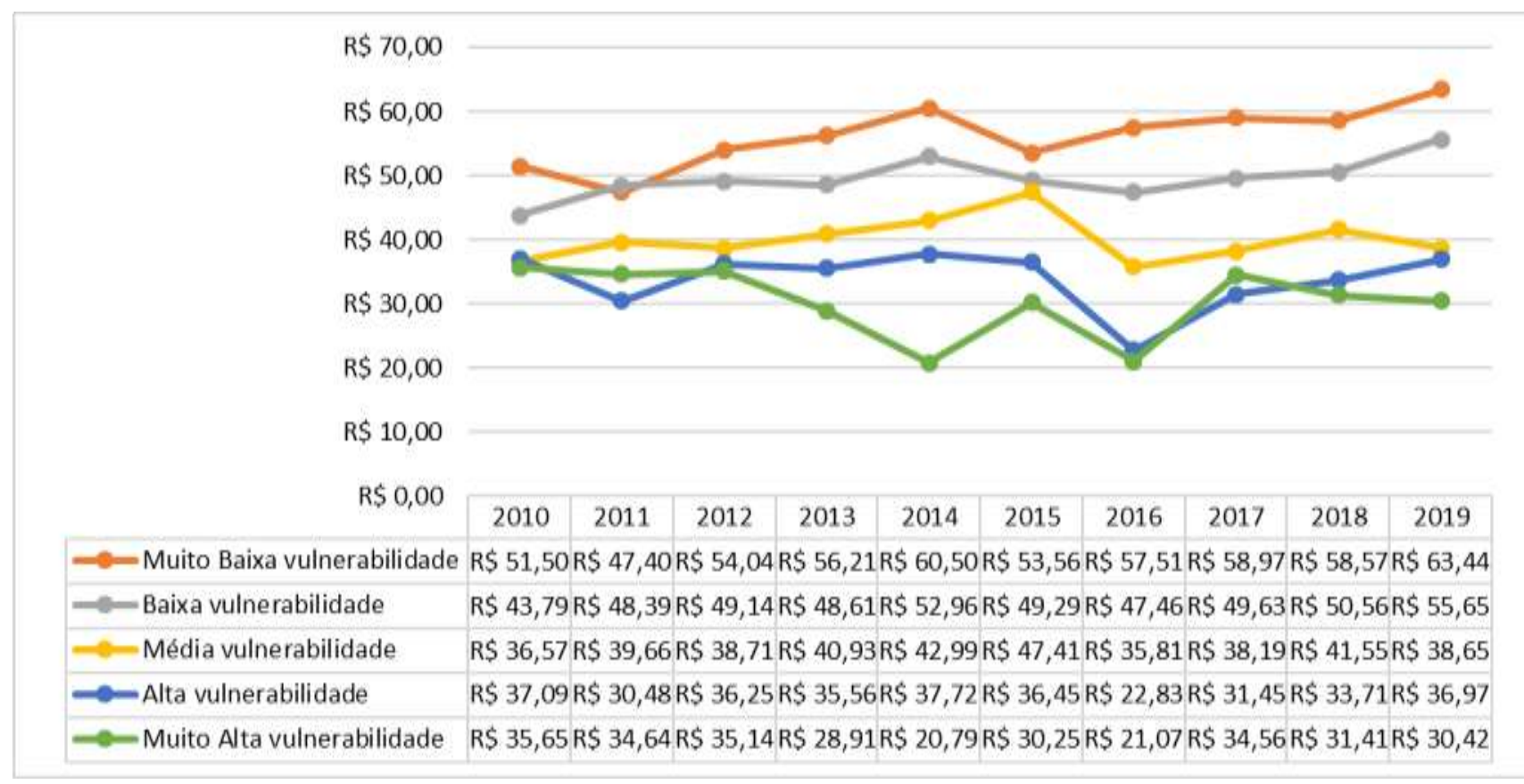

Valores corrigidos para janeiro de 2020 pelo Índice de Preços ao Consumidor Amplo - IPCA. Fonte: Autores (2021).

Pelo gráfico acima, pode-se observar que quanto maior a vulnerabilidade social do município, menores são os valores investidos em medicamentos e que os municípios que apresentam muito alta vulnerabilidade mostram queda no valor per capta investido no intervalo (14,7\%), ao passo que os municípios de muito baixa vulnerabilidade mostram uma expansão de $23,2 \%$ nesse valor.

\section{Discussão}

No cenário de crise econômica e processo de desfinanciamento do SUS, sabe-se que a discussão não se restringe a somente incrementar os recursos para esse sistema, mas implica, sobretudo, aprimorar a forma de distribuição dos recursos da União para os estados e municípios, aperfeiçoando os critérios de rateio, conforme estabelecidos pela Lei no 141/2012 (Mendes et al., 2020). Esta discussão precisa se estender também ao modelo de financiamento dos medicamentos para atenção básica, medicamentos estes que constituem recurso fundamental para a resolutividade da atenção à saúde, modelo não tratado nesse artigo que trata de financiamento dos medicamentos em geral. Pelos dados levantados nesse estudo observa-se que os investimentos per capta em saúde cresceram nos municípios, em que se observa um aumento de 33,9\% nesse valor, também cresceu por parte da União, em que se tem um incremento de 30,7\%, ao passo que os estados investiram menos $0,87 \% \mathrm{em}$ saúde. Isso mostra que os municípios brasileiros estão aumentando seus investimentos em saúde mais que os demais entes. Araújo et al. (2017) mostram que a ampliação da participação dos municípios no financiamento do Sistema Único de Saúde, por meio da vinculação de recursos mínimos de arrecadação de cada ente federativo em Ações e Serviços Públicos de Saúde (ASPS), converteu-se em uma das principais alternativas para solucionar, ou mesmo remediar, o subfinanciamento do SUS. No 
caso dos municípios, isso se sucedeu à custa de grandes desequilíbrios orçamentários, dado o comprometimento de grande parte dos seus orçamentos com o setor.

Já o comportamento dos investimentos em medicamento foi diferente do identificado em saúde, pois observa-se também um aumento nos investimentos da União $(23,5 \%)$, porém bem maior que dos municípios $(1,61 \%)$ e dos estados, que apresentaram queda nesse investimento (-69,2\%). Vieira (2019) já comenta que entre 2010 e 2017, a participação federal no financiamento do gasto em medicamentos do SUS passou de 71\% para 85\%. Em 2019, pelos dados aqui levantados, essa porcentagem foi de $79 \%$. Os últimos anos foram marcados pela centralização da compra de medicamentos no Ministério da Saúde e responsabilização do órgão pela aquisição da maior parte dos novos medicamentos incorporados, como antineoplásicos e os imunossupressores (Luz et al., 2017), além de programas como o Farmácia Popular do Brasil. No entanto, observa-se um crescimento menor nos investimentos da União em medicamentos que nos investimentos em saúde (30,7\%) entre 2012 e 2019.

De acordo com dados de 161 Estados Membros da OMS de 1995 a 2006, os gastos per capita com produtos farmacêuticos aumentaram em aproximadamente 50\%, e esses aumentos foram mais pronunciados nos países de renda média, onde os gastos farmacêuticos em 2006 foram 1,76 vezes maiores do que em 1995 (World Health Organization, 2011). Apesar do fato dos medicamentos representarem um dos custos de maior e mais rápido crescimento para os sistemas de saúde em todo o mundo, o que se observou com os dados aqui levantados em relação aos municípios, assim como a União, foi que houve no Brasil um aumento maior nos investimentos em saúde (32,8\%) que nos investimentos em medicamentos (1,61\%). Este fenômeno, no período estudado, no Brasil, chama a atenção para a necessidade de um olhar mais atencioso para os investimentos nesse item (medicamento) e para a investigação das causas desse fenômeno e suas consequências para a qualidade e resolutividade da saúde da população brasileira.

Já os estados diminuíram seus investimentos per capta em medicamentos em $69,2 \%$, uma queda bem maior que a observada nos investimentos em saúde por esses entes. Bruns et al. (2014) evidenciam que dentre os fatores que dificultam a efetivação das ações sistemáticas da Assistência Farmacêutica está a falta de contrapartida da Secretaria Estadual de Saúde para o Programa Farmácia Básica, constatada em 20\% dos municípios paraibanos. De forma agravante ao fator financiamento, é uma queixa comum entre os gestores municipais de assistência farmacêutica em alguns estados a falta de apoio, de qualquer ordem, por parte dos gestores estaduais (Barreto e Guimarães, 2010; Mendes et al, 2016).

Foi observado que em 2019 os municípios da pesquisa gastaram em média no Brasil R \$ 45,09 com medicamentos adquiridos para seus munícipes, um valor bem acima da soma das contrapartidas do Componente Básico da Assistência Farmacêutica (CBAF), que pode chegar no máximo a apenas $\mathrm{R} \$ 10,77$, valor verificado para os municípios com os menores IDHM (Ministério da Saúde, 2019). Além disso, a desigualdade de investimentos em medicamentos é flagrante, tendo os municípios com mais alta vulnerabilidade chegando a investir $\mathrm{R} \$ 30,00 \mathrm{em}$ média. Entre as regiões, as disparidades também são claras, com os municípios das regiões Norte e Nordeste com os menores investimentos em saúde, R\$701,50 e R\$736,40, e em medicamentos $R \$ 23,58$ e $R \$ 24,20$, respectivamente.

Apesar da maior aplicação de recurso pelos municípios observado nesse estudo, Pontes et al. (2017) e Oliveira et al. (2018) observaram que, em relação ao CBAF, alguns municípios não utilizam o recurso das contrapartidas federal e estadual na sua totalidade, o que pode refletir na necessidade de investimento em medicamentos em outros componentes. Oliveira et al. (2018) mostraram que apesar dos responsáveis pela Assistência Farmacêutica dos municípios participantes da sua pesquisa terem referido insuficiência de recursos para aquisição de medicamentos $(68,4 \%)$ e a não aplicação do total pactuado para o CBAF pelos entes no ano de 2013 (21,1\%), os recursos orçados para aquisição de medicamentos também não foram utilizados em sua totalidade no ano anterior em cerca de um terço dos municípios, apontando fragilidade no processo de gestão da Assistência Farmacêutica no estado da região Nordeste. 
Pontes et al. (2017) já observaram que a grande maioria dos municípios (73\%) aplicou, na compra de medicamentos para a atenção básica (considerando apenas os da RENAME), um valor por habitante/ano menor do que o mínimo recomendado pela legislação vigente. Verificaram, também, que a região Norte é a que possui maior número de municípios com menor aplicação de recursos e que os da região Sudeste, em média, aplicaram um valor por habitante/ano maior e adquiriram mais itens do que aqueles das demais regiões.

Essas assimetrias não são, de forma alguma, restritas aos investimentos financeiros. Diversos aspectos da assistência farmacêutica municipal avaliados pela Pesquisa Nacional sobre Acesso e Uso Racional de medicamentos (PNAUM), apresentaram problemas maiores nas regiões Norte e Nordeste, como infraestrutura e capacidade de gestão (Leite, Silvana Nair et al., 2017; Gerlack et al., 2017). Nestas duas regiões é onde se concentram os municípios com menores taxas de farmacêuticos contratados pelo serviço público por habitante (Faraco et al., 2020). Como consequência, a disponibilidade de medicamentos da atenção básica também é menor nos municípios destas mesmas regiões (Nascimento et al., 2017).

Os impactos de tais assimetrias de investimento em saúde e em medicamentos certamente são contundentes para a saúde dos munícipes. Álvares, et al., (2017) identificaram que a maior proporção dos pacientes que declararam ter recebido totalmente os medicamentos de que necessitavam eram na região Sul $(48,1 \%)$ e a menor na região Norte $(37 \%)$. No entanto, o cenário de investimentos dos municípios é ainda mais agravante na atualidade pois, em 2019, dados da Pesquisa Nacional de Saúde revelaram que apenas 30,5\% das pessoas obtiveram seus medicamentos no SUS, na média nacional, indicando retração da acessibilidade na atenção básica.

Nas situações que se observa a ampliação do gasto em medicamentos não significa necessariamente ampliação da cobertura populacional, porque pode ocorrer concentração de despesas para poucas pessoas, o que pode acontecer em razão da judicialização da saúde e de outros fatores. Como também pode ocorrer concentração do investimento em produtos de alto preço, podendo ser decorrente de um processo ainda deficitário de avaliação da incorporação tecnológica e de regulação econômica do mercado (Vieira, 2019).

Da mesma forma, nas situações em que se observa uma redução do gasto não implica obrigatoriamente diminuição da cobertura, pois os preços de alguns dos produtos podem ter reduzido; a relação de medicamentos pode ter sido modificada de um ano para outro, sem prejuízo para a terapêutica; e os protocolos de utilização de medicamentos, assim como outras medidas para racionalizar o uso dos medicamentos podem ter sido implementados, corroborando para melhor eficiência na utilização dos recursos. Dessa forma, investigações sobre os fatores que expliquem a diminuição ou o aumento do gasto necessitam ser realizadas, assim como estudos para a identificar as causas da mudança desses fatores (Vieira, 2019).

Corrobora com tudo levantado na pesquisa a informação que as regiões que mais investiram em saúde e medicamentos foram justamente as que apresentam IVS melhor que a média nacional $(0,351)$, ou seja, região Centro-Oeste $(0,299)$, Sudeste $(0,287)$ e Sul $(0,245)$, inclusive possuindo o município menos vulnerável da amostra, Boa Vista do Buricá/RS (Região Sul). Segundo Mendes et al. (2020), um financiamento redistributivo e compromissos reforçados com a equidade e o acesso universal à saúde fazem os sistemas de saúde reduzirem as desigualdades sociais e protegerem as famílias mais vulneráveis.

Essa situação identificada no artigo não constitui novidade para o histórico quadro de desigualdade social no país, pois Costa et al. (2016) já haviam demonstrado que a maior proporção dos residentes nas regiões economicamente mais vulneráveis do país, Norte (IVS 0,469) e Nordeste (IVS 0,454), tiveram que arcar, em 2011, com os custos dos medicamentos para hipertensão e diabetes mellitus, comparados aos das regiões mais desenvolvidas. Drummond (2020) também constatou que nas regiões com menos investimento em medicamentos observa-se uma maior prevalência de não adesão à farmacoterapia.

Pelos dados aqui levantados confirma-se a relevância do SUS para promover o acesso aos medicamentos, porém sugere-se que os governantes deem maior atenção ao investimento em medicamentos para atendimento à população, pois, 
barreiras ao acesso a medicamentos podem ter um resultado negativo a adesão ao tratamento. Como citado por Cunha \& Carnut (2012) "a disponibilidade de medicamentos é vista como um elemento primordial em um sistema de saúde de qualidade, representando um indicativo de credibilidade dos serviços e ações em saúde".

Atingir a cobertura universal de saúde para medicamentos reduz as despesas domésticas com custos de saúde, litígios de saúde, atendimento ambulatorial, hospitalização e mortalidade.

Sugere-se ainda que sejam desenvolvidas políticas públicas que diminuam as assimetrias na evolução dos investimento em saúde e medicamentos por todos os entes e que contribuam de forma a favorecer a equidade de investimentos em saúde e em medicamentos em todas as regiões do país, pois como afirmado por Mendes et al. (2020), o acúmulo de evidências e a literatura internacional também sugerem que a crise econômica e a recessão contemporânea constituem ameaça à saúde e aos cuidados de saúde das populações porque a recessão econômica e políticas restritivas como respostas à crise efeitos indiretos para a saúde - podem reduzir o acesso aos cuidados de saúde, especialmente para grupos populacionais vulneráveis, e aumentar as disparidades no acesso a cuidados de saúde diante das quedas nas receitas públicas direcionadas para o sistema de saúde, bem como reduções na prestação de seus serviços.

Além do já exposto aqui, Vieira (2019) cita que os elevados preços dos novos medicamentos geram grandes desafios aos sistemas de saúde em todo o mundo, independentemente da renda do país.

\section{Considerações Finais}

Este trabalho indicou assimetrias regionais nos investimentos em saúde e medicamentos no Brasil, além da diferente evolução desses investimentos no período analisado, sendo as regiões mais vulneráveis as que menos investiram, e mostrou ainda que os investimentos em saúde não estão sendo acompanhados dos investimentos em medicamentos.

As informações levantadas aqui, mesmo que muito uteis, mas pelo objetivo proposto, não esclarecem questões sobre os fatores determinantes do comportamento dos gastos no intervalo da pesquisa, o que deixa a sugestão para outros estudos nesse sentido.

As análises realizadas nesse trabalho podem propiciar subsídios para o redirecionamento de políticas públicas, com o objetivo de favorecer a promoção de ações de saúde e de Assistência Farmacêutica mais equânimes no país. Os resultados sinalizam ainda a necessidade da realização de pesquisas sobre os investimentos em medicamentos e na organização dos serviços de Assistência Farmacêutica no Brasil, visando à melhor compreensão a respeito das barreiras ao acesso a medicamentos e até mesmo à serviços de saúde e das assimetrias existentes para combater o grande desafio que é reduzir as desigualdades regionais de investimento em saúde e medicamentos no Brasil.

Dessa forma, propomos trabalhos no intuito de identificar os fatores que corroboram para esse comportamento das assimetrias regionais nos investimentos em saúde e medicamentos identificados no trabalho e que proponham políticas de saúde que levem aos investimentos em medicamentos acompanhar os investimentos em saúde.

\section{Referências}

Álvares, J., et al. (2017). Acesso aos medicamentos pelos usuários da atenção primária no Sistema Único de Saúde. Rev Saude Publica, 10. https://www.scielo.br/pdf/rsp/v51s2/pt_0034-8910-rsp-S1518-51-s2-87872017051007139.pdf

Álvares, J., Alves, M. C. G. P., Escuder, M. M. L., Almeida, A. M., Izidoro, J. B., Junior, A. A. G., Costa, K. S., Costa, E. A., Guibu, I. A., Soeiro, O. M., Leite, S. N., Karnikowski, M. G. de O., \& Acurcio, F. de A. (2017). Pesquisa Nacional sobre Acesso, Utilização e Promoção do Uso Racional de Medicamentos: métodos. Revista de Saúde Pública, 51(suppl.2), 1-9. https://doi.org/10.11606/s1518-8787.201705100supl2ed

Banco Central do Brasil. (n.d.). Calculadora do Cidadão. https://www3.bcb.gov.br/CALCIDADAO/publico/exibirFormCorrecaoValo res.do?method=exibirFormCorrecaoValores\&aba=1 
Castro, M. C., Massuda, A., Almeida, G., Menezes-Filho, N. A., Andrade, M. V., de Souza Noronha, K. V. M., Rocha, R., Macinko, J., Hone, T., Tasca, R., Giovanella, L., Malik, A. M., Werneck, H., Fachini, L. A., \& Atun, R. (2019). Brazil's unified health system: the first 30 years and prospects for the future. Lancet (London, England), 394(10195), 345-356. https://doi.org/10.1016/S0140-6736(19)31243-7

Chagas, V. O., Provin, M. P., Mota, P. A. P., Guimarães, R. A., \& Amaral, R. G. (2020). Institutional strategies as a mechanism to rationalize the negative effects of the judicialization of access to medicine in Brazil. BMC Health Services Research, 20(1), 1-14. https://doi.org/10.1186/s12913-020-4929-9

Costa, K. S. (2014). Acesso e uso de medicamentos: inquéritos de saúde como estratégia de avaliação.

Costa, K. S., Francisco, P. M. S. B., Malta, D. C., \& Barros, M. B. de A. (2016). Fontes de obtenção de medicamentos para hipertensão e diabetes no Brasil: resultados de inquérito telefônico nas capitais brasileiras e no Distrito Federal, 2011 TT - Sources of medicines for hypertension and diabetes in Brazil: telephone survey results. Cad. Saúde Pública, 32(2), e00090014-e00090014. http://www.scielosp.org/scielo.php?script=sci_arttext\&pid=S0102$311 \mathrm{X} 2016000200703$

Cunha, C. L. F., \& Carnut, L. (2012). Políticas e Sistemas de Saúde no Brasil. Cadernos de Saúde Pública, 29(7), 1478-1479. https://doi.org/10.1590/S0102$311 \mathrm{X} 2013000700022$

Dias, L. L. dos S., Santos, M. A. B. dos, \& Pinto, C. D. B. S. (2019). Regulação contemporânea de preços de medicamentos no Brasil - uma análise crítica. Saúde Em Debate, 43(121), 543-558. https://doi.org/10.1590/0103-1104201912120

Drummond, E. D. (2020). Desigualdades Socioeconômicas No Acesso A Medicamentos E Na Adesão À Farmacoterapia No Brasil.

Drummond, E. D., Simões, T. C., \& de Andrade, F. B. (2020). An evaluation of non-adherence to pharmacotherapy for chronic diseases and socioeconomic inequalities in brazil. Revista Brasileira de Epidemiologia, 23, 1-14. https://doi.org/10.1590/1980-549720200080

Faraco, E. B., Guimarães, L., Anderson, C., \& Leite, S. N. (2020). The pharmacy workforce in public primary healthcare centers: Promoting access and information on medicines. Pharmacy Practice, 18(4), 1-7. https://doi.org/10.18549/PharmPract.2020.4.2048

Garcia, M. M., Azevedo, P. S., Mirelman, A., Safatle, L. P., Iunes, R., Bennie, M. C., Godman, B., \& Guerra Junior, A. A. (2020). Funding and Service Organization to Achieve Universal Health Coverage for Medicines: An Economic Evaluation of the Best Investment and Service Organization for the Brazilian Scenario. Frontiers in Pharmacology, 11, 370. https://doi.org/10.3389/fphar.2020.00370

Gerlack, L. F., Karnikowski, M. G. de O., Areda, C. A., Galato, D., Oliveira, A. G. de, Álvares, J., Leite, S. N., Costa, E. A., Guibu, I. A., Soeiro, O. M., Costa, K. S., Junior, A. A. G., \& Acurcio, F. de A. (2017). Gestão da assistência farmacêutica na atenção primária no Brasil. Rev Saude Publica, 1-11.

IPEA. (2015). Atlas da Vulnerabilidade Social nos Municípios Brasileiros. In Ipea (p. 77).

Kondro, W. (2007). Drug spending tops \$25 billion. CMAJ, 176(13), 1816-1816. https://doi.org/10.1503/CMAJ.070680

Leite, S. N.; et al (2017). Infraestrutura das farmácias da atenção básica no Sistema Único de Saúde: Análise dos dados da PNAUM-Serviços. Revista de Saúde Pública, 51(Supl), 2-13.

Luiza, V. L., Chaves, L. A., Campos, M. R., Bertoldi, A. D., Silva, R. M., Bigdeli, M., Ross-Degnan, D., \& Emmerick, I. C. M. (2018). Applying a health system perspective to the evolving Farmácia Popular medicines access programme in Brazil. BMJ Global Health, 2 (Suppl 3), e000547. https://doi.org/10.1136/bmjgh-2017-000547

Luz, T. C. B., Osorio-De-Castro, C. G. S., Magarinos-Torres, R., \& Wettermark, B. (2017). Trends in medicines procurement by the Brazilian federal government from 2006 to 2013. PLoS ONE, 12(4), 1-13. https://doi.org/10.1371/journal.pone.0174616

Massuda, A., Hone, T., Leles, F. A. G., Castro, M. C. de, \& Atun, R. (2018). The Brazilian health system at crossroads: progress, crisis and resilience. BMJ Global Health, 3(4), e000829. https://doi.org/10.1136/BMJGH-2018-000829

Mendes, Á., Leite, M. G., \& Carnut, L. (2020). Uma metodologia para rateio dos recursos federais do SUS: o índice de necessidades de saúde. Rev Saude Publica, 54-77.

Ministério da Saúde. (2017). SIOPS. https://antigo.saude.gov.br/repasses-financeiros/siops/indicadores

Ministério da Saúde. (2019). Portaria GM n ${ }^{\circ} 3.193$, de 9 de dezembro de 2019. Altera a Portaria de Consolidação no 6/GM/MS, de 28 de setembro de 2017, para dispor sobre o financiamento do Componente Básico da Assistência Farmacêutica no âmbito do Sistema Único de Saúde (SUS). Diário Oficial Da União.

Nascimento, R. C. R. M. do, J. Álvares, A. Guerra Junior, Gomes, I. C., Silveira, M. R., Costa, E. A., Leite, S. N., Costa, K. S., Soeiro, O. M., Guibu, I. A., Karnikowski, M. G. de O., \& Acurcio, F. de A. (2017). Polifarmácia: uma realidade na atenção primária do Sistema Único de Saúde. Revista de Saúde Pública, 51(suppl.2). https://doi.org/10.11606/S1518-8787.2017051007136

Oliveira, M. A., Luiza, V. L., Tavares, N. U. L., Mengue, S. S., Arrais, P. S. D., Farias, M. R., Pizzol, T. da S. D., Ramos, L. R., \& Bertoldi, A. D. (2016). Acesso a medicamentos para doenças crônicas no Brasil: uma abordagem multidimensional. Revista de Saude Publica, 50(supl 2), 1-13. https://doi.org/10.1590/S1518-8787.2016050006161

Paim, J., Travassos, C., Almeida, C., Bahia, L., \& MacInko, J. (2011). The Brazilian health system: History, advances, and challenges. The Lancet, 377(9779), 1778-1797. https://doi.org/10.1016/S0140-6736(11)60054-8

Reveiz, L., Chapman, E., Torres, R., Fitzgerald, J. F., Mendoza, A., Bolis, M., \& Salgado, O. (2013). Right-to-health litigation in three Latin American countries: a systematic literature review. Rev Panam Salud Publica, 33(3), 213-222. https://doi.org/10.7441/joc.2014.03.01

Santos Neto, J. A. dos, Mendes, Á. N., Pereira, A. C., Paranhos, L. R., Santos Neto, J. A. dos, Mendes, Á. N., Pereira, A. C., \& Paranhos, L. R. (2017). Análise do financiamento e gasto do Sistema Único de Saúde dos municípios da região de saúde Rota dos Bandeirantes do estado de São Paulo, Brasil. Ciência \& Saúde Coletiva, 22(4), 1269-1280. https://doi.org/10.1590/1413-81232017224.28452016 
Research, Society and Development, v. 10, n. 13, e179101320896, 2021

(CC BY 4.0) | ISSN 2525-3409 | DOI: http://dx.doi.org/10.33448/rsd-v10i13.20896

Vargas-Pelaez, C. M., Rover, M. R. M., Soares, L., Blatt, C. R., Mantel-Teeuwisse, A. K., Rossi, F. A., Restrepo, L. G., Latorre, M. C., López, J. J., Bürgin, M. T., Silva, C., Leite, S. N., \& Farias, M. R. (2019). Judicialization of access to medicines infour Latin American countries: acomparative qualitative analysis. International Journal for Equity in Health, 18(1), 68.

Vieira, F. S. (2018). Evolução do gasto com medicamentos do Sistema Único De Saúde no período de 2010 a 2016. In Rio de Janeiro. http://www.ipea.gov.br/portal/images/stories/PDFs/TDs/180117_td_2356.pdf

Vieira, F. S. (2019). Indutores do gasto direto do ministério da saúde em medicamentos (2010-2019).

Vieira, F. S., \& Zucchi, P. (2011). Aplicações diretas para aquisição de medicamentos no Sistema Único de Saúde. Revista de Saúde Pública, 45(5), 906-913. https://doi.org/10.1590/S0034-89102011005000048

Wang, D. W. L., Vasconcelos, N. P. de, Oliveira, V. E. de, \& Terrazas, F. V. (2014). Os impactos da judicialização da saúde no município de São Paulo: gasto público e organização federativa. Revista de Administração Pública, 48(5), 1191-1206. https://doi.org/10.1590/0034-76121666

World Health Organization. (2011). The World Medicines Situation 2011: Medicine expenditures ( $3^{\mathrm{a}}$ ). 